\title{
Efectos Compañero en Contextos Escolares Altamente Segregados
}

\author{
Peer Effects in Highly Segregated School Contexts
}

\author{
Natalia Krüger * \\ IIESS, Departamento de Economía, Universidad Nacional del Sur - CONICET, Argentina
}

Los sistemas educativos latinoamericanos se posicionan entre los más segregados del mundo: los grupos de estudiantes provenientes de distinto contexto social se distribuyen de forma desigual entre sus escuelas, con escasa interacción entre sí. Empleando datos de PISA 2018, el trabajo evalúa la existencia de efectos compañero sobre el desarrollo de competencias en las áreas de Lectura, Matemática y Ciencia y sobre la expectativa ocupacional de los alumnos de 15 años de edad en la región. Para ello, se estiman modelos de regresión multinivel multivariados. Se constata la presencia de efectos significativos de la composición socioeconómica escolar sobre dichos resultados, lo cual permite afirmar que la situación de segregación tiende a reforzar la inequidad educativa y social. Esto es así porque los alumnos de origen social más desfavorecido suelen asistir a escuelas cuyo alumnado se encuentra en similares condiciones, potenciando su desventaja inicial. Lo contrario ocurre para aquellos de mayor nivel socioeconómico. La evidencia aportada se encuentra en línea con los antecedentes disponibles para América Latina, contribuyendo a visibilizar el fenómeno de la segregación social escolar como un problema central de sus sistemas educativos. Se insta a las autoridades y a toda la comunidad educativa a pensar y consensuar estrategias para mitigarlo.

Descriptores: Efectos composicionales; Segregación escolar; Desigualdad de oportunidades; Escuela secundaria; América Latina.

Latin American education systems are among the most segregated in the world: since the distribution of students from different social backgrounds across schools is uneven, they scarcely interact with each other. Drawing on PISA 2018 data, this study evaluates the existence of peer effects on the development of Reading, Mathematics and Science competences and on the occupational expectation of 15year-old students in the region. To this end, it presents the estimation of multivariate multilevel regression models. Results confirm the presence of school socioeconomic composition effects on these outcomes, which allows the conclusion that the situation of segregation tends to reinforce educational and social inequality. The reason is that students from a vulnerable background are likely to attend schools with a similar socioeconomic intake, strengthening their initial disadvantage. The opposite occurs for students from a more favorable social origin. Findings are in line with previous research in Latin America, highlighting the phenomenon of socioeconomic school segregation as a central problem in these education systems. School system authorities and the entire educational community are urged to develop and agree on strategies to mitigate it.

Keywords: Composition effects; School segregation; Inequality of opportunities; Secondary education; Latin America.

*Contacto: natalia.kruger@uns.edu.ar

Recibido: 25 de marzo 2020

$1^{\text {a }}$ Evaluación: 22 de junio 2020

ISSN: 1696-4713

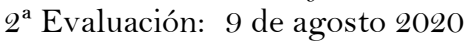

www.rinace.net/reice/

Aceptado: 16 de agosto 2020

revistas.uam.es/reice 


\section{Introducción}

La gran desigualdad social y económica que caracteriza a las sociedades latinoamericanas (Alvaredo y Gasparini, 2015) se ve reflejada en sus sistemas educativos, los cuales se ubican entre los más segregados por nivel socioeconómico. Distintos estudios aportan evidencia que indica que, si bien la intensidad y la evolución del fenómeno difieren entre los países de la región, en general los niveles de segregación social escolar son altos, y superan a los de otras regiones con diverso grado de desarrollo (Arcidiácono et al., 2014; Krüger, 2019; Murillo, 2016; Murillo, Duk y Martínez-Garrido, 2018; Vázquez, 2016). Los últimos datos del Programa para la Evaluación Internacional de Alumnos (PISA, por sus siglas en inglés), del año 2018, confirman la vigencia de este diagnóstico: las escuelas latinoamericanas presentan los mayores niveles de aislamiento social y la más baja diversidad entre los países participantes del estudio (OCDE, 2019b).

La segregación escolar tiene efectos esperados negativos, tanto sobre la riqueza de la experiencia educativa como sobre la cohesión social a futuro, los cuales por sí mismos justificarían una preocupación por el problema. A esto se suma un potencial impacto sobre la equidad educativa: en OCDE (2019b), se observa que los países que presentan mayores niveles de segregación escolar, entre ellos los latinoamericanos, también registran una asociación más fuerte entre el estatus socioeconómico y el desempeño. El alumnado de la región logra en general peores y más desiguales resultados que el de la OCDE, con altos niveles de variación entre las escuelas.

Los canales mediante los cuales la segregación escolar puede reforzar la desigualdad de logros educativos son diversos, y se vinculan con la manera en que la composición socioeconómica incide en el desempeño individual. En forma directa, puede actuar el denominado "efecto compañero" o "efecto de pares" (Rumberger y Palardy, 2005; Schindler Rangvid, 2007; Van Ewijk y Sleegers, 2010), el cual hace referencia a la influencia que la interacción con los compañeros puede tener en la conducta, las actitudes y la motivación de cada alumno, al contribuir a generar un contexto más o menos propicio para el trabajo escolar. En forma indirecta, el perfil socioeconómico del alumnado puede impactar mediante su asociación con otros factores escolares. Las características de la población estudiantil suelen vincularse con la forma de trabajo y las expectativas de los docentes, con el currículum, con la cantidad y calidad de los recursos materiales, con el nivel de participación de los padres, con el estilo de gestión, entre otros (Bellei, 2013; Palardy, 2013; Schindler Rangvid, 2007; Willms, 2006).

Entonces, directa o indirectamente, la existencia de efectos composicionales significativos en un sistema educativo altamente segregado redundará en una profundización de las desventajas para los jóvenes más vulnerables y de las ventajas para quienes provienen de un contexto favorecido (Bonal y Bellei, 2018; Dupriez y Dumay, 2006; Mickelson, 2018). Es decir, el impacto del origen social de los alumnos se verá potenciado por la muy probable asistencia a una escuela con un perfil socioeconómico homogéneo y similar al propio.

El objetivo general del presente artículo es proveer evidencia actualizada para América Latina acerca de la existencia de efectos significativos de la composición socioeconómica de las escuelas en los resultados educativos. Ahora bien, dichos resultados son múltiples y de muy variada índole: en la escuela no sólo se adquieren conocimientos y aptitudes en las distintas áreas de enseñanza sino que también se desarrollan actitudes, valores, hábitos 
y expectativas (Bloom, 1956; Delors, 1996; Dreeben, 1968; Krathwohl et al., 1964). Los primeros, pueden denominarse resultados "cognitivos", mientras que los últimos corresponderían a los "no-cognitivos" (Brunello y Schlotter, 2011).

Los atributos no-cognitivos son el foco de un creciente interés en la literatura, y distintos estudios abogan por su papel clave en el desarrollo de trayectorias educativas y laborales exitosas, así como en la incorporación activa y responsable en la vida social (Brunello y Schlotter, 2011; Durlak et al., 2011; Heckman y Rubinstein, 2001; Heckman, Stixrud y Urzua, 2006; Holmlund y Silva, 2009; Levin, 2012). De hecho, se ha hallado que las características psicológicas de la personalidad inciden en los aprendizajes y los resultados de los exámenes, por lo cual resulta complejo separar los distintos tipos de competencias (Borghans, Meijers y Ter Weel, 2008; Borghans et al., 2011; Duckworth et al., 2011). Asimismo, se ha sostenido que, lejos de ser aspectos inmutables de la personalidad, las habilidades blandas podrían ser maleables y susceptibles de ser afectadas por el contexto familiar y escolar (Formichella y Krüger, 2017; Heckman, Stixrud y Urzua, 2006; Heckman et al., 2010).

Así, resulta de interés analizar no sólo la influencia de la composición socioeconómica escolar sobre los tradicionales logros cognitivos, sino también su efecto sobre los nocognitivos. Como explican Borghans y otros (2011), estos últimos son difíciles de medir y en la literatura puede encontrarse un amplio abanico de ejemplos, como las autopercepciones, la motivación, la perseverancia, las competencias sociales, la autonomía y las expectativas sobre el futuro (Brunello y Schlotter, 2011; Heckman y Rubinstein, 2001; Kautz et al., 2014; Morrison y Schoon, 2013). En particular, el presente estudio se focaliza en la expectativa de los alumnos acerca de su estatus ocupacional en el futuro. La misma puede retroalimentarse con el rendimiento escolar, el compromiso y la motivación y, como se sostiene en OCDE (2019b), puede ser muy relevante en la perpetuación de las desigualdades existentes en el mercado laboral. Las expectativas ocupacionales son probablemente un reflejo de lo que los alumnos observan en su entorno cercano y, si bien se asocian al contexto socioeconómico familiar ${ }^{1}$, este factor puede ser mediado por el contexto escolar.

Específicamente, entonces, el presente estudio analiza la significatividad de los efectos composicionales sobre distintos resultados cognitivos -el rendimiento en las áreas de Lectura, Ciencias y Matemática- y un resultado no-cognitivo -la expectativa ocupacionalde los alumnos latinoamericanos de nivel medio.

Se emplean para ello los datos de la ronda 2018 de PISA -en la que participaron Argentina, Brasil, Chile, Colombia, Costa Rica, México, Panamá, Perú, República Dominicana y Uruguay- y se estiman modelos de regresión multinivel multivariados.

\section{Revisión de la literatura}

Existe una vasta literatura internacional que ha indagado en las influencias de la composición escolar sobre los resultados educativos. Teóricamente, se han aportado

\footnotetext{
${ }^{1}$ En la tabla II.B1.6.1 de OCDE (2019b), pueden observarse amplias diferencias en la jerarquía ocupacional esperada entre los alumnos pertenecientes al primer y último cuartil de estatus socioeconómico.
} 
diversas explicaciones para la existencia de dichos efectos, los cuales pueden canalizarse a través del ambiente de aprendizaje o de la interacción entre pares. El contexto escolar es propicio para la internalización de normas y valores educativos que influyen en los comportamientos, expectativas y logros individuales. Asistir a una institución cuyo alumnado proviene de un origen social favorecido, con un mayor clima educativo en el hogar, podría contribuir a transmitir hábitos de estudio o modelos que promuevan las aspiraciones educativas, o incluso afectar las percepciones y prácticas de los docentes. La cultura escolar, la presión del entorno y los padres, la fijación de estándares de rendimiento, la competencia, la cooperación o el tutelaje entre pares, la calidad de los intercambios en clase, la disciplina o el clima escolar, representan potenciales vías a través de los cuales opera el efecto composicional (Lugo, 2011; Palardy, 2013; Schindler Rangvid, 2007; Van Ewijk y Sleegers, 2010).

Empíricamente, si bien la existencia de efectos compañero positivos ha sido en algunos casos cuestionada, la mayor parte de la evidencia parece actualmente sostenerla (Mickelson, 2018; OCDE, 2019b; Van Ewijk y Sleegers, 2010). No sólo se ha hallado un efecto de las características de la población estudiantil sobre los logros cognitivos, sino que también se han encontrado impactos sobre otros resultados académicos y no académicos de corto y largo plazo -como el disfrutar de la escuela, el compromiso con el trabajo escolar, la terminalidad en distintos niveles, las relaciones entre grupos sociales, las aspiraciones ocupacionales, las condiciones laborales, los ingresos o la probabilidad de cometer crímenes-(Ackert, 2017; Mickelson, 2018).

En América Latina, los estudios empíricos vinculados con la segregación escolar y sus potenciales consecuencias son más recientes y escasos, pero aun así existen antecedentes que apuntan en la misma dirección: los niveles importantes de segregación escolar en la región y la presencia de efectos composicionales significativos se conjugan para profundizar la desigualdad de resultados educativos, tanto en el nivel primario como en el secundario.

Algunos de dichos estudios se han enfocado específicamente en analizar los efectos composicionales sobre la adquisición de conocimientos y competencias cognitivas, mientras que otros han incluido al nivel socioeconómico escolar como variable de control en análisis más generales. Entre los trabajos que abarcan varios países latinoamericanos, cabe mencionar a Cervini (2012); Cervini, Dari y Quiroz (2016), Delprato, Köseleci y Antequera (2015), Duarte, Bos y Moreno (2009), Treviño y otros (2010), y Willms y Somers (2001). Con base en datos de pruebas internacionales estandarizadas como el Primer Estudio Internacional Comparativo (PEIC), el Segundo/Tercer Estudio Regional Comparativo y Explicativo (SERCE/TERCE) o PISA, en general estiman modelos multinivel, hallando un efecto significativo y positivo de la composición socioeconómica escolar. Vale destacar en este caso el trabajo de Delprato, Köseleci y Antequera (2015), por ser el antecedente más cercano al presente estudio. Utilizando datos de PISA 2000 y 2012 para seis países latinoamericanos (Argentina, Brasil, Chile, México, Perú y Uruguay) estiman, mediante modelos multinivel, la probabilidad de alcanzar y/o superar el nivel 1 en las tres pruebas aplicadas. Encuentran que dicho resultado presenta una alta variabilidad entre escuelas en cada país, y que más de un tercio de dicha variabilidad podría explicarse por el perfil socioeconómico diferencial de los establecimientos.

Por otro lado, hay artículos que ofrecen evidencia de efectos composicionales significativos para países individuales, como: Cervini (2003), Krüger (2018) y Marchionni, Pinto y 
Vazquez (2013) para Argentina; Bartholo y Da Costa (2015) y Firpo, Jales y Pinto (2015) para Brasil; McEwan (2003) para Chile; Hernández Padilla y Bazán Ramírez (2016) para México, o Benavides, León y Etesse (2014) para Perú.

Con respecto a la existencia de efectos compañero sobre resultados de tipo no-cognitivo, no se hallan estudios que aborden específicamente el tema en la región. Sí pueden mencionarse algunos trabajos que analizan en forma general los determinantes de otros resultados académicos y no académicos. Un estudio pionero en este sentido fue el de Cervini (2003), con datos de alumnos que se encontraban en 1998 finalizando el nivel secundario en Argentina. El autor analiza los efectos del tipo de administración escolar sobre el desempeño en Matemática y Lengua y logros no-cognitivos como las actitudes hacia las Matemáticas, la aspiración educativa y la expectativa de éxito futuro. Halla que, dada la segmentación institucional existente, al controlar por la composición socioeconómica escolar disminuye o incluso desaparece la diferencia a favor del sector privado en los distintos resultados. Krüger y Formichella (2019), por su parte, emplean datos de PISA 2012 para Argentina y estiman un modelo de ecuaciones estructurales para analizar si las competencias no-cognitivas pueden actuar como mediadoras entre los factores explicativos tradicionales y los logros cognitivos. Encuentran que la composición social escolar incide tanto directamente en el rendimiento académico como indirectamente a través del compromiso con la escuela. En cambio, en un trabajo anterior y utilizando los mismos datos (Formichella y Krüger, 2017), estiman simultáneamente los determinantes de la nota en Matemáticas y de un resultado no-cognitivo, la apertura a la resolución de problemas, hallando efectos compañero significativos sólo para la primera variable.

En suma, los antecedentes disponibles permiten hipotetizar que existe un efecto significativo y positivo de la composición socioeconómica escolar en los distintos tipos de logros educativos en los países latinoamericanos. Sin embargo, la literatura que aborda esta temática aún es escasa en la región, y vale la pena aportar evidencia adicional para contrastar dicha hipótesis. Como se especificará más adelante, este estudio se diferencia de los previos al incluir información más actualizada y para una mayor cantidad de países latinoamericanos; y al analizar simultáneamente el efecto composicional sobre distintas competencias cognitivas y no-cognitivas.

\section{Método}

\subsection{Datos}

Se emplea la ronda 2018 de PISA, el estudio mundial implementado por la Organización para la Cooperación y el Desarrollo Económicos (OCDE), al cual se han ido incorporando progresivamente los países latinoamericanos. La población objetivo de PISA es aquella que tiene entre 15 años y 3 meses y 16 años y 2 meses al momento de la prueba, y que se encuentra escolarizada cursando al menos el séptimo año. Las muestras del estudio son, en cada país, representativas para este sub-conjunto de los jóvenes. Las mismas surgen de un proceso en dos etapas en el que se selecciona en primer lugar un conjunto de al menos 150 escuelas en forma estratificada; luego, en cada una de ellas se selecciona al azar una muestra de aproximadamente 42 alumnos². El tamaño muestral para los países

\footnotetext{
${ }^{2}$ La información detallada sobre la población y la muestra puede encontrarse en OCDE (2019a).
} 
latinoamericanos participantes en el 2018 se encuentra entre 5000 y 12000 alumnos (cuadro 1). PISA busca evaluar mediante pruebas estandarizadas los conocimientos y habilidades de los jóvenes para participar en la vida económica y social. Se examinan las competencias del alumnado en tres áreas principales: Lectura, Matemática y Ciencia, enfatizándose una de ellas en cada ronda. En el año 2018, el foco fue puesto en Lectura (OCDE, 2019a). A su vez, se administran cuestionarios complementarios al alumnado y las autoridades escolares, los cuales permiten caracterizar el contexto familiar y escolar, así como conocer distintos atributos personales de los estudiantes.

\subsection{Variables}

Variables dependientes:

- Lectura: el puntaje obtenido en dicha prueba pretende reflejar la capacidad del alumnado para comprender y trabajar con textos y así poder alcanzar sus objetivos y desarrollar su potencial.

- Matemática: el puntaje obtenido dicha en prueba indica la capacidad para utilizar las matemáticas en distintas situaciones, aplicando la lógica matemática y utilizando procedimientos y conceptos de dicha índole.

- Ciencias: el puntaje obtenido en dicha prueba busca mostrar la capacidad para trabajar con cuestiones vinculadas con las ciencias, explicando los fenómenos científicamente, diseñando preguntas científicas e interpretando evidencia.

Los valores para cada competencia se ubican en un rango de 0 a 1000, con una media de 500 y un desvío estándar de 100 para los países de la OCDE (OCDE, 2019a). No se registran valores perdidos para ninguna de las tres variables entre los países latinoamericanos participantes. Cabe aclarar que PISA reporta los puntajes obtenidos por cada alumno en las pruebas en forma de diez valores plausibles extraídos de su probable distribución de habilidades, la cual se estima a posteriori a través de un modelo que incorpora las repuestas a todos los ítems de los exámenes, así como las variables de los cuestionarios de contexto. Se ha seguido aquí el método recomendado por la OCDE (2017), que implica estimar los parámetros de interés a través del promedio de los valores obtenidos luego de estimar los modelos para cada conjunto de valores plausibles.

- Expectativa ocupacional: el estatus ocupacional esperado es el índice BSMJ elaborado por PISA, que surge de preguntar al alumnado acerca de la ocupación que esperan tener a la edad de 30 años. Las respuestas son codificadas y mapeadas empleando la metodología de Ganzeboom y Treiman (2003). Cuanto mayor es el valor del índice, más alta es la jerarquía ocupacional esperada (OCDE, 2017). Al igual que otros índices del proyecto PISA, los valores son estandarizados para tener una media de 0 y un desvío estándar de 1 para la OCDE. Valores negativos del índice, entonces, indican que el alumno o la alumna espera tener una ocupación de menor jerarquía que la esperada en promedio por el alumnado de dichos países. Esta variable presenta una tasa de respuesta promedio del $75 \%$ entre los países latinoamericanos participantes del operativo $^{3}$.

\footnotetext{
${ }^{3}$ Argentina (78\%); Brasil (71\%); Chile (76\%); Colombia (85\%); Costa Rica (83\%); México (76\%); Rep. Dominicana (59\%); Panamá (65\%); Perú (75\%); Uruguay (75\%).
} 
Variables explicativas:

- Perfil socioeconómico escolar: La principal variable de interés para el estudio es la composición socioeconómica escolar, estimada aquí a través del promedio escolar del índice de estatus económico, social y cultural (ESCS) elaborado por PISA. El mismo es derivado de las respuestas del alumnado acerca del nivel educativo y el estatus ocupacional de sus padres, así como de las posesiones del hogar, que son un proxy para el nivel de riqueza. Posee una media de o y un desvío estándar de 1 entre los participantes de la OCDE (OCDE, 2019b).

Variables de control ${ }^{4}$ :

Fueron escogidas con la intención de incluir un conjunto completo de factores de control, los cuales han sido reconocidas por la literatura especializada como determinantes significativos del rendimiento escolar y de distintos resultados no-cognitivos. A su vez, pretenden minimizar los posibles sesgos de selección derivados de la asignación no aleatoria del alumnado a las escuelas (Krüger, 2018).

- Edad.

- Género.

- Apoyo emocional (emosups): índice del apoyo emocional que brindan los padres en relación a los esfuerzos, logros y dificultades en la escuela.

- Asistencia al preescolar: indica si el alumnado asistió al nivel inicial a la edad de 4 años o antes, o bien si nunca asistió o lo hizo a partir de los 5 años.

- Nivel socioeconómico del hogar: índice ESCS del alumno.

- Asistencia al grado modal: indica si el alumnado asiste al grado modal para su país o se encuentra rezagado.

- Proporción de mujeres en la escuela.

- Tamaño escolar (schsize): es la cantidad total de alumnos en la escuela.

- Tipo de gestión escolar: indica si la escuela es de gestión estatal o privada.

- Localización: distingue a las escuelas ubicadas en un pequeño pueblo (con 15000 habitantes o menos) de aquellas ubicadas en una ciudad o pueblo con una población mayor.

- Escasez de recursos educativos (edushort): índice que registra si la escuela presenta escasos o inadecuados recursos materiales e infraestructura.

- Escasez de personal docente (staffshort): indica la percepción de la autoridad escolar acerca de la falta de personal docente y auxiliar y de la adecuación de su calificación.

- Interés del personal docente: indica la percepción de los estudiantes acerca del entusiasmo presentado por los docentes del área de Lengua.

- Proporción de docentes con título de Maestría.

4. En OCDE (2019b) se describen en mayor detalle los índices construidos a partir de los cuestionarios de contex to de PISA. 
- Agrupamiento intra-escolar: refleja si en el establecimiento los estudiantes son agrupados por habilidad en distintas clases para una o más materias.

- Selectividad escolar: indica si en la escuela siempre se consideran los antecedentes académicos o los resultados de un examen para la admisión de los alumnos o bien si se consideran algunas veces o nunca.

Cabe aclarar que se realizaron los siguientes procesos previos al análisis: i) se excluyeron aquellas escuelas con una muestra igual o inferior a cinco alumnos, para una mejor estimación de la composición escolar, ii) se centraron las variables explicativas cuantitativas en su media global, y iii) se imputaron los valores perdidos de las variables explicativas a través del algoritmo EM, un método de máxima verosimilitud (De Leeuw, Meijer y Goldstein, 2008) ${ }^{5}$.

El tamaño de la muestra en cada país y la descripción de las principales variables empleadas en el estudio pueden encontrarse en el cuadro 1.

Cuadro 1. Tamaño muestral y descripción de las variables principales

\begin{tabular}{|c|c|c|c|c|c|c|c|c|c|c|c|}
\hline & \multirow[t]{2}{*}{$\mathbf{N}$} & \multicolumn{2}{|c|}{$\begin{array}{l}\text { Nota EN } \\
\text { LECTURA }\end{array}$} & \multicolumn{2}{|c|}{$\begin{array}{l}\text { NoTA EN } \\
\text { MATEMÁT. }\end{array}$} & \multicolumn{2}{|c|}{$\begin{array}{l}\text { Nota EN } \\
\text { CiEnCIAS }\end{array}$} & \multicolumn{2}{|c|}{$\begin{array}{l}\text { EXPECT. } \\
\text { OCUPACI. }\end{array}$} & \multicolumn{2}{|c|}{$\begin{array}{c}\text { COMP. } \\
\text { SOCIAL } \\
\text { ESCOLAR }\end{array}$} \\
\hline & & $\mathbf{M}$ & D.E. & $\mathbf{M}$ & D.E. & $\mathbf{M}$ & D.E. & M & D.E. & M & D.E. \\
\hline Argentina & 11.975 & 402 & 98 & 379 & 84 & 404 & 90 & 69,5 & 16,7 & $-0,957$ & 0,751 \\
\hline Brasil & 10.691 & 413 & 100 & 384 & 88 & 404 & 90 & 72,2 & 16,7 & $-1,108$ & 0,812 \\
\hline Chile & 7.621 & 452 & 92 & 417 & 85 & 444 & 83 & 70,0 & 17,8 & $-0,586$ & 0,700 \\
\hline Colombia & 7.522 & 412 & 89 & 391 & 81 & 413 & 82 & 72,8 & 14,6 & $-1,187$ & 0,826 \\
\hline C. Rica & 7.221 & 426 & 81 & 402 & 75 & 416 & 73 & 74,6 & 14,2 & $-0,962$ & 0,833 \\
\hline México & 7.299 & 420 & 84 & 409 & 78 & 419 & 74 & 73,2 & 13,6 & $-1,182$ & 0,870 \\
\hline Panamá & 6.270 & 377 & 88 & 353 & 77 & 365 & 85 & 69,3 & 14,7 & $-1,104$ & 0,887 \\
\hline Perú & 6.086 & 401 & 92 & 400 & 84 & 404 & 80 & 69,8 & 17,1 & $-1,120$ & 0,858 \\
\hline R. Dominicana & 5.674 & 342 & 82 & 325 & 71 & 336 & 71 & 74,7 & 14,1 & $-1,068$ & 0,609 \\
\hline Uruguay & 5.263 & 427 & 96 & 418 & 85 & 426 & 87 & 68,3 & 19,2 & $-0,986$ & 0,724 \\
\hline
\end{tabular}

Nota: N: cantidad de alumnos en la muestra original; D.E.: desviación estándar; Composición social escolar: índice ESCS promedio por escuela.

Fuente: Elaboración propia a partir de la base de datos PISA 2018 (OCDE).

\subsection{Enfoque metodológico}

Se emplea una estrategia metodológica cuantitativa encuadrada en la tradición de la función de producción educativa para el análisis estadístico de los determinantes del aprendizaje escolar (Hanushek, 1979). Siguiendo a la literatura especializada, se estima un modelo de regresión multinivel que se adecua a la estructura jerárquica de los datos alumnos agrupados en escuelas- y permite obtener estimadores más eficientes (De Leeuw, Meijer y Goldstein, 2008; Hox, 2002). Estos modelos posibilitan la descomposición de la varianza del resultado educativo, para conocer si las desigualdades se observan

\footnotetext{
${ }^{5}$ Se empleó el comando mi impute mon de Stata, el cual utiliza un método de Monte Carlo vía cadenas de Markov. Se incluyó un conjunto completo de variables personales, familiares y escolares, el cual puede consultarse a la autora.
} 
principalmente entre establecimientos o bien entre alumnos en su interior. De este análisis se obtiene el Coeficiente de Correlación Intraclase (CII), que indica la proporción de la varianza total correspondiente al nivel escuela (muestra en qué medida la desigualdad en los resultados globales surge de las desigualdades registradas entre las escuelas, lo cual refleja el grado de diferenciación horizontal del sistema educativo).

Adicionalmente, siendo el objetivo evaluar los efectos compañero en distintos tipos de resultados, es apropiado estimar modelos multinivel multivariados ${ }^{6}$, que reconocen la posible interdependencia entre las variables explicadas. Las notas obtenidas por un mismo alumno para las distintas competencias cognitivas probablemente se encuentren relacionadas entre sí, así como con sus expectativas educativas y laborales. Al estimar los determinantes de cada resultado en forma simultánea a través de un único sistema de ecuaciones, se minimizan los sesgos y se obtienen pruebas de significatividad más confiables (Rasbash et al., 2012; Snijders y Bosker, 1999).

Se estiman dos modelos: i) por un lado, un modelo trivariado que intenta explicar la nota obtenida en las tres competencias evaluadas por PISA, y ii) por otro lado, un modelo bivariado que busca explicar la nota en Lectura junto con la expectativa de carrera. Cabe aclarar que si bien se reconoce que los determinantes pueden diferir en ambos casos, se emplea el mismo modelo para alcanzar una mayor comparabilidad.

Siguiendo la estrategia usual, se estiman inicialmente los modelos nulos que permiten descomponer la varianza, y luego los modelos que incorporan variables explicativas, para conocer la significatividad de sus coeficientes y la proporción de la varianza que logran explicar. Se emplea para ello el comando mixed del programa Stata 14. Las ecuaciones que especifican la forma funcional de los modelos estimados se presentan en el Anexo 2.

\section{Resultados}

\subsection{Modelo trivariado}

El primer resultado de interés surge de la estimación del modelo nulo y del cálculo de los CCI para cada país y competencia (cuadro 2). La proporción de la varianza observada entre escuelas es en promedio del $45 \%$ para la prueba de Lectura, del 44\% para Matemática y del 43\% para Ciencias. Aún en el caso de México, donde los valores del CCI son menores, se observa que los logros del alumnado difieren significativamente entre establecimientos educativos. Esto puede deberse tanto a los perfiles diferenciados de su población estudiantil como a distintos atributos escolares, y puede considerarse un primer indicio de la segmentación horizontal de los sistemas educativos. Junto con los resultados del test de máxima verosimilitud aplicado, estos datos confirman la pertinencia de estimar modelos de tipo multinivel. A su vez, cabe mencionar que las covarianzas entre las tres variables dependientes son positivas y significativas al $1 \%$ en todos los países, lo cual constata la conveniencia de estimar en forma simultánea los determinantes de cada competencia.

${ }^{6}$ Una explicación formal detallada de los modelos multinivel multivariados puede encontrarse en Formichella y Krüger (2017). 
Cuadro 2. Regresión Multinivel. CCI y varianza explicada por los modelos finales: modelos trivariados

\begin{tabular}{|c|c|c|c|c|c|c|c|c|c|c|c|c|c|}
\hline & \multirow{2}{*}{$\mathbf{N}$} & \multicolumn{4}{|c|}{ NOTA EN LECTURA } & \multicolumn{4}{|c|}{ Nota EN MATEMÁTICA } & \multicolumn{4}{|c|}{ Nota EN CiEnCias } \\
\hline & & CCI & $(1)$ & $(2)$ & (3) & CCI & $(1)$ & $(2)$ & (3) & CCI & $(1)$ & $(2)$ & (3) \\
\hline Argentina & 11.952 & 42,8 & 79,9 & 11,0 & 40,6 & 44,0 & 82,7 & 11,0 & 42,6 & 41,4 & 79,6 & 10,3 & 39,0 \\
\hline Brasil & 10.531 & 47,8 & 69,8 & 8,1 & 53,5 & 48,0 & 68,6 & 8,0 & 37,1 & 48,1 & 69,3 & 8,5 & 37,7 \\
\hline Chile & 7.549 & 47,1 & 88,1 & 9,6 & 46,5 & 50,7 & 88,1 & 10,4 & 49,7 & 47,4 & 86,8 & 8,3 & 45,6 \\
\hline Colombia & 7.471 & 46,2 & 71,8 & 13,8 & 40,6 & 44,0 & 67,6 & 14,9 & 38,1 & 42,4 & 70,2 & 11,4 & 36,4 \\
\hline C. Rica & 7.216 & 45,1 & 82,6 & 9,8 & 42,6 & 43,8 & 77,6 & 12,2 & 40,8 & 46,1 & 81,4 & 11,0 & 43,5 \\
\hline México & 7.192 & 36,2 & 60,1 & 2,0 & 23,1 & 33,2 & 53,8 & 3,2 & 20,0 & 32,6 & 58,0 & 2,4 & 20,5 \\
\hline Panamá & 6.133 & 51,0 & 73,7 & 3,8 & 39,5 & 47,5 & 75,1 & 5,4 & 38,5 & 49,0 & 69,7 & 3,8 & 36,1 \\
\hline Perú & 5.937 & 46,0 & 75,7 & 8,9 & 39,6 & 41,8 & 74,1 & 13,5 & 38,9 & 43,0 & 72,7 & 11,4 & 37,7 \\
\hline R. Dom. & 5.582 & 43,3 & 76,2 & 14,2 & 41,0 & 40,4 & 76,9 & 10,4 & 37,2 & 38,2 & 75,1 & 11,8 & 36,0 \\
\hline Uruguay & 5.219 & 45,4 & 86,3 & 13,6 & 46,7 & 45,1 & 88,5 & 15,3 & 48,3 & 43,0 & 85,9 & 13,2 & 44,5 \\
\hline
\end{tabular}

Nota: N: cantidad de observaciones válidas en la especificación final; (1) Porcentaje de la varianza residual explicado por las variables sobre el modelo nulo: nivel escuelas;

(2) Porcentaje de la varianza residual explicado por las variables sobre el modelo nulo: nivel alumnos; (3) Porcentaje de la varianza residual explicado por las variables sobre el modelo nulo: total.

Fuente: Elaboración propia a partir de la base de datos PISA 2018 (OCDE). 
El cuadro 2 también permite observar que luego de la incorporación de todas las variables de control en la especificación preferida se puede explicar un porcentaje relevante, cercano al $75 \%$ en promedio, de la varianza entre establecimientos para cada competencia (1). En general, al igual que en los antecedentes previos al estudio, dar cuenta de la variación de los resultados escolares al interior de las escuelas es más complejo, y la proporción de la varianza que aún resta explicar es alta, cercana al 90\% (2). Sin embargo, siendo tan relevante en la región latinoamericana la desigualdad de los resultados verificada entre las escuelas, los modelos finales dan cuenta, en promedio, de un $38 \%$ a un $41 \%$ de la varianza en el rendimiento en las distintas pruebas (3).

En gran medida, esto se debe a la alta capacidad explicativa de algunas variables incorporadas en el análisis. En el cuadro 3 se presentan los resultados para la variable independiente de interés en este estudio: la composición socioeconómica escolar (los resultados para el conjunto completo de variables se encuentran en el cuadro 6 del Anexo $1)$.

Cuadro 3. Regresión multinivel. Coeficientes de la variable "composición socioeconómica escolar" en los modelos finales: modelos trivariados

\begin{tabular}{|c|c|c|c|c|c|c|}
\hline & \multicolumn{2}{|c|}{ NOTA EN LECTURA } & \multicolumn{2}{|c|}{ NOTA EN MATEMÁTICA } & \multicolumn{2}{|c|}{ Nota En CiEncias } \\
\hline & Coef. & Coef. est. & Coef. & Coef. est. & Coef. & Coef. est. \\
\hline Argentina & $41,24 * * *$ & 0,316 & $38,24 * * *$ & 0,341 & 37,39 *** & 0,312 \\
\hline Brasil & $35,19^{* * *}$ & 0,286 & $29,05^{* * *}$ & 0,272 & $29,38 * * *$ & 0,262 \\
\hline Chile & $41,36 * * *$ & 0,315 & $39,26^{* * *}$ & 0,328 & $37,79^{* * * *}$ & 0,308 \\
\hline Colombia & $38,45^{* * * *}$ & 0,359 & $34,33 * * *$ & 0,351 & $35,95^{* * * *}$ & 0,361 \\
\hline C. Rica & $39,40 * * *$ & 0,402 & $35,97 * * *$ & 0,404 & 34,21 *** & 0,395 \\
\hline México & $16,27^{*}$ & 0,169 & 10,08 & 0,113 & $16,18^{*}$ & 0,189 \\
\hline Panamá & $29,11^{* * * *}$ & 0,295 & $27,12^{* * *}$ & 0,311 & $26,75^{* * * *}$ & 0,275 \\
\hline Perú & $35,77 * * *$ & 0,334 & $28,05^{* * *} *$ & 0,287 & $26,90^{* * * *}$ & 0,292 \\
\hline R. Dominicana & $28,92 * * *$ & 0,216 & $25,38 * * *$ & 0,220 & $22,55^{* * * *}$ & 0,194 \\
\hline Uruguay & $31,52^{* * * *}$ & 0,237 & $28,57 * * *$ & 0,240 & $25,58 * * *$ & 0,212 \\
\hline
\end{tabular}

Nota: Coef.: valor del coeficiente; Coef. est.: valor del coeficiente estandarizado. ${ }^{* * *}$ Significatividad al $1 \%$.

Fuente: Elaboración propia a partir de la base de datos PISA 2018 (OCDE).

Puede observarse que en todos los casos -con la excepción de Matemática en México-, aun controlando por un conjunto completo de variables personales, familiares y escolares, el nivel socioeconómico promedio de la escuela tiene un impacto esperado positivo y significativo en el rendimiento individual. Concretamente, para un alumno promedio, un incremento de 1 punto en el índice de composición escolar permite esperar un incremento aproximado de entre 28 y 42 puntos en la nota de Lectura, de entre 25 y 40 puntos en la nota de Matemática y de entre 22 y 38 puntos en la nota en Ciencias, según el país y exceptuando a México donde los efectos son menores. Estos puntajes representan aproximadamente entre un $6 \%$ y un $10 \%$ de los promedios nacionales en las distintas pruebas. Asimismo, para facilitar la interpretación, puede tenerse en cuenta que un incremento de 1 punto en el índice de composición escolar sería equivalente a pasar, por ejemplo, del percentil 25 al 75 de su distribución en cada país. Alternativamente, un incremento de 1 desvío estándar en dicho índice permite esperar un incremento de entre 0,21 y 0,40 desvíos estándar en el rendimiento en Lectura, de entre 0,22 y 0,35 desvíos estándar en el rendimiento en Matemática, y de entre 0,19 y 0,40 desvíos estándar en el 
rendimiento en Ciencias. Estos resultados sugieren entonces que el perfil socioeconómico de los compañeros de escuela influye en los conocimientos y habilidades que puede alcanzar un alumno, tal como son captados por las pruebas de PISA.

De hecho, los valores de los coeficientes para el factor de interés se encuentran entre los más altos del conjunto de variables explicativas, apenas superados en algunos casos por la asistencia al grado modal, la proporción de mujeres o la formación de posgrado de los docentes (cuadro 6 del Anexo 1). Con respecto al efecto de las variables de control, si bien hay ciertas variaciones entre los resultados para cada país, en general los factores incorporados tienen los signos esperados. Por ejemplo, el haber asistido al nivel inicial es un aspecto que incide positivamente en el desempeño, así como el provenir de un hogar con un mayor nivel socioeconómico. A su vez, el máximo coeficiente es registrado para la asistencia al grado modal, variable que condensa la acumulación de distintos factores personales y familiares que favorecen el rendimiento. El ser mujer, por su parte, permite esperar un mejor resultado para Lectura, pero peor para Matemática y Ciencias. Las variables de nivel escuela resultan mayormente no significativas, con algunas excepciones, como la proporción de mujeres, el interés de los docentes, el agrupamiento por habilidad y la formación de los docentes, con signos que en algunos casos difieren entre países y competencias.

A modo de ejemplo y para comparar con el efecto de la composición escolar, puede mencionarse que las brechas de género representan en promedio 0,15 desvíos estándar de los puntajes en las distintas pruebas; que la asistencia al grado modal o al preescolar permite en promedio esperar un incremento de 0,56 y de 0,05 desvíos estándar en los resultados, respectivamente; y que el título de Maestría de los docentes aporta en promedio un rendimiento adicional esperado de 0,13 desvíos estándar, siendo significativo solo en algunos países.

\subsection{Modelo bivariado}

Para evaluar el efecto de la composición socioeconómica escolar en las expectativas de carrera del alumnado minimizando la complejidad de los modelos, se estiman sus determinantes en forma simultánea solo con la principal competencia cognitiva examinada en PISA 2018. Los resultados para la Nota en Lectura confirman los obtenidos a partir de los modelos trivariados, por lo que vale la pena concentrarse aquí en los resultados para la expectativa ocupacional.

El cuadro 4 presenta los CCI para esta variable, los cuales resultan mucho menores a los correspondientes a los logros cognitivos, pero aún son significativos y relevantes. En promedio, cerca de un $11 \%$ de las desigualdades en la expectativa ocupacional se explican por diferencias al nivel de las escuelas, y los tests aplicados verifican la conveniencia de estimar modelos multinivel. A su vez, la covarianza entre la nota en Lectura y la expectativa de carrera es positiva y significativa en todos los casos, lo que amerita su estimación simultánea.

Nuevamente, la proporción de la varianza explicada por los modelos finales a nivel de escuelas es alta, en promedio cercana al 50\%, con una mayor variabilidad entre los países (1). En el caso de la República Dominicana, por ejemplo, el modelo completo solo explica un $7 \%$ de la varianza entre escuelas, mientras que en el caso de Chile la cifra es superior al $80 \%$. De cualquier forma, la capacidad explicativa total en este caso resulta bastante 
menor que para los resultados cognitivos, y solo en Brasil, Chile y Uruguay se logra explicar más del $10 \%$ de la varianza total (3).

Cuadro 4. Regresión multinivel. CCI y varianza explicada por los modelos finales: Modelos bivariados

\begin{tabular}{lccccccccc}
\hline & \multirow{2}{*}{$\mathbf{N}$} & \multicolumn{3}{c}{ NOTA EN LECTURA } & \multicolumn{4}{c}{ EXPECTATIVA OCUPACIONAL } \\
\cline { 3 - 9 } & & CCI & $(\mathbf{1})$ & $(\mathbf{2})$ & $\mathbf{( 3 )}$ & CCI & $(\mathbf{1})$ & $\mathbf{( 2 )}$ & $\mathbf{( 3 )}$ \\
\hline Argentina & 9.310 & 42,5 & 81,4 & 10,7 & 40,5 & 7,1 & 52,5 & 6,0 & 9,0 \\
Brasil & 7.540 & 48,4 & 58,2 & 8,0 & 32,3 & 12,1 & 45,0 & 7,1 & 11,7 \\
Chile & 5.771 & 46,0 & 89,1 & 9,8 & 46,3 & 22,1 & 81,2 & 5,3 & 22,0 \\
Colombia & 6.356 & 43,0 & 72,4 & 14,3 & 39,3 & 4,4 & 36,9 & 2,7 & 4,3 \\
C. Rica & 5.965 & 44,0 & 82,6 & 11,1 & 42,7 & 7,9 & 60,7 & 3,2 & 7,7 \\
México & 5.485 & 34,7 & 63,9 & 2,5 & 23,8 & 8,9 & 42,1 & 1,6 & 5,2 \\
Panamá & 3.981 & 50,7 & 76,3 & 3,8 & 40,5 & 12,8 & 35,9 & 1,9 & 6,3 \\
Perú & 4.478 & 44,7 & 78,3 & 8,6 & 39,8 & 13,5 & 45,2 & 4,3 & 9,8 \\
R. Dominicana & 3.343 & 41,3 & 77,2 & 14,4 & 40,4 & 3,2 & 7,2 & 3,2 & 3,4 \\
Uruguay & 3.907 & 45,0 & 87,8 & 14,8 & 47,7 & 22,5 & 83,2 & 10,6 & 26,9 \\
\hline
\end{tabular}

Notas: N: cantidad de observaciones válidas en la especificación final; (1) Porcentaje de la varianza residual explicado por las variables sobre el modelo nulo: nivel escuelas; (2) Porcentaje de la varianza residual explicado por las variables sobre el modelo nulo: nivel alumnos; (3) Porcentaje de la varianza residual explicado por las variables sobre el modelo nulo: total.

Fuente: Elaboración propia a partir de la base de datos PISA 2018 (OCDE).

La estimación de los modelos finales, con el mismo conjunto de variables de control que en el caso de los modelos trivariados, muestra que en seis de los diez países latinoamericanos participantes de PISA, la composición social escolar incide positiva y significativamente en la ocupación esperada a futuro por el alumnado (cuadro 5). Los efectos no parecen ser tan fuertes como en el caso del rendimiento en las pruebas, ya que un incremento de 1 punto en el índice de composición escolar, cuando resulta significativo, permite esperar un incremento aproximado de entre 1 y 3,5 puntos en el índice de expectativa ocupacional, valores que representan del $1,5 \%$ al $3,5 \%$ de los promedios nacionales para dicho índice. Asimismo, un aumento de 1 desvío estándar en la composición escolar permite esperar un incremento de entre 0,05 y 0,14 desvíos estándar en el índice de expectativa ocupacional. De todas formas, la composición socioeconómica escolar es una de las escasas variables con capacidad explicativa sobre dicho resultado (cuadro 7 del Anexo 1).

En estos modelos, los efectos y la significatividad de las variables de control son dispares, siendo el género uno de los factores más relevantes, con un coeficiente positivo para el caso de las mujeres. También resultan significativos y positivos el asistir al grado modal y el nivel socioeconómico del hogar; y solo en algunos casos también la edad, el tipo de gestión escolar y otras variables relativas al centro educativo. A fin de evaluar la magnitud relativa de los distintos efectos, puede mencionarse que la brecha a favor de las mujeres y de quienes asisten al grado modal es en promedio de 0,3 y 0,22 desvíos estándar del índice de expectativa ocupacional, respectivamente. Asimismo, el promedio de los coeficientes estandarizados del NSE individual es de 0,067 .

${ }^{7}$ Estos datos pueden ser solicitados a la autora. 
Cuadro 5. Regresión Multinivel. Coeficientes de la variable "composición socioeconómica escolar" en los modelos finales: modelos bivariados

\begin{tabular}{|c|c|c|c|c|}
\hline & \multicolumn{2}{|c|}{ NOTA EN LECTURA } & \multicolumn{2}{|c|}{ EXPECTATIVA OCUPACIONAL } \\
\hline & Coef. & Coef. est. & Coef. & Coef. est. \\
\hline Argentina & $42,28 * * *$ & 0,324 & $1,14^{*}$ & 0,050 \\
\hline Brasil & $36,66^{* * *}$ & 0,298 & $1,16^{*}$ & 0,056 \\
\hline Chile & $41,49 * * *$ & 0,316 & $3,78 * * *$ & 0,143 \\
\hline Colombia & $39,70^{* * *}$ & 0,371 & $1,14^{* *}$ & 0,064 \\
\hline C. Rica & $39,07 * * *$ & 0,398 & $2,22 * * *$ & 0,132 \\
\hline México & $17,20 * *$ & 0,179 & $0,19^{\mathrm{ns}}$ & 0,012 \\
\hline Panamá & $29,60 * * *$ & 0,300 & $-0,27 \mathrm{~ns}$ & $-0,016$ \\
\hline Perú & $33,74 * * *$ & 0,315 & $1,08^{\mathrm{ns}}$ & 0,054 \\
\hline R. Dominicana & $29,87 * * *$ & 0,223 & $0,91^{\mathrm{ns}}$ & 0,039 \\
\hline Uruguay & $31,89 * * *$ & 0,240 & $3,50 * * *$ & 0,132 \\
\hline
\end{tabular}

\section{Discusión y conclusiones}

El trabajo ha verificado que una proporción relevante de la desigualdad en distintos resultados cognitivos y no-cognitivos de los alumnos de 15 años de edad participantes del estudio PISA 2018 en América Latina se origina entre establecimientos educativos. Esto se explica porque las escuelas difieren tanto en el perfil de su alumnado como en otras características, como recursos y procesos. En este marco, el principal resultado del trabajo es que en la mayoría de los países existe un efecto significativo de la composición socioeconómica escolar tanto en el desempeño en Lectura, Matemática y Ciencias como en la expectativa de carrera, la cual puede influir en los logros laborales futuros.

En general, explicar las desigualdades en los resultados de tipo no-cognitivo resulta más complejo que dar cuenta de la varianza en los resultados cognitivos, lo cual es consistente con antecedentes como Cervini (2003) y Formichella y Krüger (2017). Son escasos los determinantes usualmente contemplados por la literatura especializada que en este caso evidencian un efecto sobre la expectativa ocupacional. Esto otorga mayor importancia relativa al hallazgo de que la composición escolar tendría un efecto positivo y significativo en la mayoría de los países considerados. Si bien queda para futuros estudios indagar en por qué su efecto es irrelevante en algunos países, vale la pena explicitar que este resultado no parece estar relacionado con las disparidades en las tasas de respuesta de la variable dependiente, ni con un efecto relativo muy diferente de las demás variables explicativas.

Por otro lado, cabe aclarar que aquí no se ha hecho una distinción entre un efecto neto o estricto del nivel socioeconómico de los pares y un efecto indirecto o conjunto que puede surgir de su interacción con otros factores escolares. Si la distribución desigual de los alumnos se da en un marco más general de segmentación horizontal en el sistema educativo, en el que los recursos humanos y materiales, las prácticas docentes y los estilos de gestión también difieren entre centros, los efectos de la variable composicional podrían estar operando conjuntamente con los de los demás factores (Dumay y Dupriez, 2008; Krüger, 2018). De hecho, en (OCDE, 2019b) se constata que en los países latinoamericanos participantes, las escuelas en ventaja -cuyos alumnos en promedio pertenecen al último cuartil del índice ESCS- y en desventaja -cuyos alumnos en 
promedio pertenecen al primer cuartil del índice ESCS- difieren en las cualificaciones y el nivel de ausentismo de sus docentes, así como en la cantidad y calidad de la infraestructura y los recursos materiales.

De cualquier forma, lo importante es comprobar que, en contextos educativos altamente segregados, el establecimiento al que asiste un joven tiene un efecto determinante en sus resultados esperados, los cuales pueden exceder al ámbito académico. Puede afirmarse entonces que los alumnos provenientes de contextos familiares vulnerables se ven expuestos a un doble riesgo educativo (Willms, 2003): por un lado, su origen social dificulta su desempeño exitoso en la escuela; por el otro, tienen una alta probabilidad de asistir a un centro con un alumnado en similares condiciones, donde los efectos de pares son desfavorables, lo cual implica un impacto negativo adicional en sus logros. La situación contraria se espera para aquellos alumnos de mayor estatus socioeconómico, quienes en general pueden beneficiarse tanto de sus propias condiciones favorables para el estudio como de efectos de pares positivos.

La elección escolar se vuelve entonces una decisión crucial para las familias, lo cual genera incentivos que tienden a profundizar la segregación, en sociedades marcadas por la fragmentación socioeconómica, cultural y simbólica (Krüger, 2019). Asimismo, constatar que la segregación social escolar contribuye a profundizar las desigualdades educativas y, potencialmente, las laborales y sociales posteriores, convierte al fenómeno en un problema relevante para la política educativa.

En este sentido, el presente trabajo aporta evidencia nueva que permite confirmar los escasos hallazgos previos para distintos países latinoamericanos. A su vez, el estudio incorpora algunos elementos novedosos en relación a los antecedentes, que otorgan robustez a los resultados y exploran nuevos aspectos del tema. Por un lado, se emplea la información más actual disponible hasta el momento incorporando nuevos países latinoamericanos; se definen especificaciones diferentes de los modelos en términos de las variables dependientes e independientes escogidas; y se realiza una estimación simultánea de los distintos resultados, lo cual resulta pertinente considerando su interdependencia. Por otro lado, se incursiona en el análisis del impacto de la composición socioeconómica estudiantil en resultados que exceden lo estrictamente cognitivo o académico, para destacar que las actitudes, motivación y expectativas de los alumnos pueden verse afectadas, con potenciales efectos sobre la movilidad social y la equidad en un sentido más amplio.

Ahora bien, no puede soslayarse que pese a haber empleado herramientas que presentan un alto nivel de aceptación en la literatura internacional -la estimación de una función de producción educativa a través de modelos de regresión multinivel con base en datos del programa PISA-, el estudio no se encuentra exento de limitaciones. Por un lado, el método no permite descartar totalmente la presencia de sesgos por la asignación no aleatoria de los alumnos a sus escuelas, aunque los mismos se han intentado minimizar incorporando un conjunto completo de controles para captar posibles características no observables. Por otro lado, la base de datos presenta algunas deficiencias que cabe mencionar: i) por definición, quedan fuera de la población objetivo aquellos alumnos que se encuentran más rezagados o directamente excluidos del sistema, lo cual puede llevar a subestimar los efectos composicionales cuando los niveles de acceso son bajos y/o el rezago es alto; ii) si bien provee información acerca de resultados de tipo no-cognitivo, permitiendo considerar un conjunto más amplio de logros educativos, la tasa de no respuesta para estos 
indicadores es relativamente alta; y iii) no permite evaluar los efectos acumulativos de los factores explicativos en el tiempo. Finalmente, debe reconocerse la dificultad de medir los resultados no-cognitivos y de separarlos de los logros cognitivos, así como de otros incentivos y características personales. Como indican Borghans y otros (2011), los resultados en pruebas pueden ser en realidad un reflejo de distintos aspectos de la personalidad, lo cual vuelve muy desafiante la tarea de identificar los atributos o logros de interés. A su vez, las medidas de habilidades no-cognitivas derivadas del auto-reporte pueden verse distorsionadas, ya que quienes responden pueden tener distintos estándares o puntos de referencia al interpretar las preguntas (Kautz et al., 2014) o bien responder lo que consideran socialmente deseable (Paulhus, 1991).

A pesar de las mencionadas limitaciones, el presente estudio contribuye a reforzar el consenso académico en torno a la relevancia de la segregación social escolar como fuente de desigualdad de oportunidades. Visibilizar este problema y concientizar a la comunidad educativa, las autoridades y la sociedad en general sobre sus consecuencias, es un primer paso clave para avanzar en el diseño de políticas orientadas a mitigarlo. Repensar entre todos los procesos de asignación de los alumnos, así como la distribución de aquellos factores escolares que se asocian estrechamente a las características del alumnado, podría reportar beneficios sustanciales en términos de reducir la inequidad. Para ello, es importante también continuar indagando en las causas de la situación actual de alta segregación, las cuales pueden ser diferentes en cada país o incluso al interior de los mismos.

Queda abierta a partir de aquí una rica agenda de investigación: por un lado, se planea avanzar en una línea orientada a conocer en profundidad los mecanismos que reducen la diversidad social de las escuelas en la región -mediante la realización de estudios de corte cualitativo- y a pensar en conjunto medidas para revertir dicho proceso; por otro lado, aún queda mucho por explorar en relación a los efectos de la composición socioeconómica sobre los resultados educativos, especialmente sobre los atributos no-cognitivos, para lo cual se continuará indagando en la forma de medición de los mismos y en su retroalimentación con los resultados cognitivos.

\section{Agradecimientos}

Se agradece el financiamiento proveniente de la SeCyT, UNS, a través del Proyecto de Grupos de Investigación (PGI) "Equidad educativa: Segmentación escolar en la localidad de Bahía Blanca"; y de la Agencia Nacional de Promoción Científica y Tecnológica a través del Proyecto de Investigación Científica y Tecnológica (PICT) 2018-02967.

\section{Referencias}

Ackert, E. (2017). Segregation paradox? School racial/ethnic and socioeconomic composition and racial/ethnic differences in engagement. Social Science Research, 70, 144-162. https://doi.org/10.1016/j.ssresearch.2017.10.010

Alvaredo, F. y Gasparini, L. (2015). Recent trends in inequality and poverty in developing countries. En R. Atkinson y C. Bourguignon (Eds.), Handbook of income distribution (pp. 321 345). Elsevier. https://doi.org/10.1016/B978-0-444-59428-0.00010-2 
Arcidiacono, M., Cruces, G., Gasparini, L., Jaume, D., Serio, M. y Vazquez, E. (2014). La segregación escolar público-privada en América Latina. CEPAL.

Bartholo, T. y Costa, M. (2016). Evidence of a school composition effect in Rio de Janeiro public schools. Ensaio: Avaliação e Políticas Públicas em Educação, 24(92), 498-521. https://doi.org/10.1590/SO104-40362016000300001

Bellei, C. (2013). El estudio de la segregación socioeconómica y académica de la educación chilena. Estudios Pedagógicos, 39(1), 325-345. https://doi.org/10.4067/S0718-07052013000100019

Benavides, M., León, J. y Etesse, M. (2014). Desigualdades educativas y segregación en el sistema peruano. Una mirada comparativa de las pruebas PISA 2000 y 2009. GRADE.

Bloom, B. (1956). A taxonomy of educational objectives: Handbook I, the cognitive domain. Longmans.

Bonal, X. y Bellei, C. (2018). Introduction: The renaissance of school segregation in a context of globalization. En X. Bonal y C. Bellei (Eds.), Understanding school segregation: Patterns, causes and consequences of spatial inequalities in education (pp. 123-154). Bloomsbury Academic. https://doi.org/10.5040/9781350033542.ch-001

Borghans, L., Meijers, H. y Ter Weel, B. (2008). The role of noncognitive skills in explaining cognitive test scores. Economic Inquiry, 46, 2-12. https://doi.org/10.1111/j.1465-7295.2007.00073.x

Borghans, L., Golsteyn, H., Heckman, J. y Humphries, J. (2011). Identification problems in personality psychology. Personality and Individual Differences, 51(3), 315-320. https://doi.org 10.1016/j.paid.2011.03.0299

Brunello, G. y Schlotter, M. (2011). Non-cognitive skills and personality traits: Labour market relevance and their development in education ङ $^{2}$ training systems. IZA

Cervini, R. (2003). Diferencias de resultados cognitivos y no cognitivos entre estudiantes de escuelas públicas y privadas en la educación secundaria de Argentina: Un análisis multinivel. Education Policy Analysis Archives, 1 1(6), 1-32.

Cervini, R. (2012). El efecto escuela en países de América Latina: Reanalizando los datos del SERCE. Education Policy Analysis Archives, 20(39), 1-35. https://doi.org/10.14507/epaa.v20n39.2012

Cervini, R., Dari, N. y Quiroz, S. (2016). Las determinaciones socioeconómicas sobre la distribución de los aprendizajes escolares. Los datos del TERCE. REICE. Revista Iberoamericana sobre Calidad, Eficacia y Cambio en Educación, 14(4), 61-79.

https://doi.org/10.15366/reice2016.14.4.003

De Leeuw, J., Meijer, E. y Goldstein, H. (2008). Handbook of multilevel analysis. Springer.

Delors, J. (1996). La educación encierra un tesoro. Informe a la UNESCO de la comisión internacional sobre la educación para el siglo XXI. UNESCO.

Delprato, N., Köseleci, N. y Antequera, G. (2015). Educación para todos en América Latina: Evolución del impacto de la desigualdad escolar en los resultados educativos. Revista Latinoamericana de Educación Comparada, 6(8), 45-75.

Dreeben, R. (1968). On what is learned in school. Addison Wesley.

Duarte, J., Bos, S. y Moreno, M. (2009). Inequidad en los aprendizajes escolares en Latinoamérica. BID.

Duckworth, A., Quinn, P., Lynam, D., Loeber, R. y Stouthamer-Loeber, M. (2011). Role of test motivation in intelligence testing. Proceedings of the National Academy of Sciences, 108(19), 7716-7720. https://doi.org/10.1073/pnas.1018601108 
Dumay, X. y Dupriez, V.(2008). Does the school composition effect matter? Evidence from Belgian data. British Journal of Educational Studies, 56(4), 440-477.

https://doi.org/10.1111/j.1467-8527.2008.00418.x

Durlak, J., Weissberg, R., Dymnicki, A., Taylor, R. y Schellinger, H. (2011). The impact of enhancing students social and emotional learning: A meta-analysis of school-based universal interventions. Child Development, 82(1), 405-432. https://doi.org/10.1111/j.1467-8624.2010.01564.x

Dupriez, V. y Dumay, X. (2006). Inequalities in school systems: Effect of school structure or of society structure? Comparative Education, 42(2), 243-260. https://doi.org/10.1080/03050060600628074

Firpo, S., Jales, H. y Pinto, C. (2015). Measuring peer effects in the Brazilian school system. Applied Economics, 47(32), 3414-3438. https://doi.org /10.1080/00036846.2015.1016207

Formichella, M. M. y Krüger, N. (2017). Reconociendo el carácter multifacético de la educación: Los determinantes de los logros cognitivos y no-cognitivos en la escuela media Argentina. El Trimestre Económico, 84(333), 165-191. https://doi.org/10.20430/ete.v84i333.266.

Ganzeboom, H. y Treiman, D. (2003). Three internationally standardised measures for comparative research on occupational status. En J. Hoffmeyer-Zlotnik y C. Wolf (Eds.), Advances in cross-national comparison, a European working book for demographic and socioeconomic variables (pp. 159-193). Kluwer Academic Press.

Hanushek, E. (1979). Conceptual and empirical issues in the estimation of educational production functions. Journal of Human Resources, 14, 351-388.

Heckman J. y Rubinstein, Y. (2001). The importance of noncognitive skills: Lessons from the GED testing program. The American Economic Review, 91(2), 145-149.

Heckman, J, Stixrud, J. y Urzua, S. (2006). The effects of cognitive and noncognitive abilities on labor market outcomes and social behavior. Journal of Labor Economics, 24(3), 411-482. https://doi.org/10.1086/504455

Heckman, J., Hyeok, S., Pinto, R., Savelyev, P. y Yavitz, A. (2010). The rate of return to the high/scope Perry Preschool program. Journal of Public Economics, 94, 114-128. https://doi.org/10.1016/j.jpubeco.2009.11.001

Hernández Padilla, E. y Bazán Ramírez, A. (2016). Efectos contextuales, socioeconómicos y culturales, sobre los resultados de México en lectura en PISA 2009. REICE. Revista Iberoamericana sobre Calidad, Eficacia y Cambio en Educación, 14(2), 79-95. https://doi.org/10.15366/reice2016.14.2.005

Hox, J. (2002). Multilevel analysis: Techniques and applications. Lawrence Erlbaurn Associates.

Kautz, T., Heckman, J., Diris, R., Ter Weel, B. y Borghans, L. (2014). Fostering and measuring skills: Improving cognitive and non-cognitive skills to promote lifetime success. OCDE.

Krathwohl, D., Bloom, B. y Masia, B. (1964). Taxonomy of educational objectives: Handbook II, the affective domain. David MacKay.

Krüger, N. (2018). An evaluation of the intensity and impacts of socioeconomic school segregation in Argentina. En X. Bonal y C. Bellei (Eds.), Understanding school segregation: Patterns, Causes and consequences of spatial inequalities in education (pp. 210-243). Bloomsbury Academic.

Krüger, N. (2019). La segregación por nivel socioeconómico como dimensión de la exclusión educativa: 15 años de evolución en América Latina. Archivos Analíticos de Políticas Educativas, 27(8), 1-23. https://doi.org/10.14507/epaa.27.3577 
Krüger, N. y Formichella, M. M. (2019). ¿Las competencias no cognitivas actúan como mediadoras en el proceso de enseñanza-aprendizaje? Evidencia para Argentina. Cuadernos de Economía, 38(77), 493-521. https://doi.org/10.15446/cuad.econ.v38n77.68582

Levin, H. (2012). More than just test scores. Prospects, Quarterly Review of Comparative Education 36(1), 61-85. https://doi.org/10.1177/1555458915626761

Lugo, M. (2011). Heterogenous peer effects, segregation and academic attainment. The World Bank.

Marchionni, M., Pinto, F. y Vazquez, E. (2013). Determinantes de la desigualdad en el desempeño educativo en la Argentina. AAEP.

McEwan, P. (2003). Peer effects on student achievement: Evidence from Chile. Economics of Education Review, 22, 131-141. https://doi.org/10.1016/S0272-7757(02)00005-5

Mickelson, R. (2018). A synthesis of social science research on the effects of ethnic, racial and socioeconomic composition of schools in the United States. En X. Bonal y C. Bellei (Eds.), Understanding school segregation: Patterns, causes and consequences of spatial inequalities in education (pp. 123-154). Bloomsbury Academic.

https://doi.org/10.5040/9781350033542.ch-007

Morrison, L. y Schoon, I. (2013). The impact of non-cognitive skills on outcomes for young people. Literature review. Institute of Education, University of London.

Murillo, F. J. (2016). Midiendo la segregación escolar en américa latina. Un análisis metodológico utilizando el TERCE. REICE. Revista Iberoamericana sobre Calidad, Eficacia y Cambio en Educación, 14(4), 33-60. https://doi.org/10.15366/reice2016.14.4.002

Murillo, F. J., Duk, C. y Martínez-Garrido, C. (2018). Evolución de la segregación socioeconómica de las escuelas de América Latina. Estudios Pedagógicos, 44(1), 157-179.

https://doi.org/10.4067/SO718-07052018000100157

OCDE. (2017). PISA 2015 technical report. OECD Publishing.

OCDE. (2019a). PISA 2018 results (volume I): What students know and can do. OECD Publishing. https://doi.org/10.1787/5fo7c754-en

OCDE. (2019b). PISA 2018 results (volume II): Where all students can succeed. OECD Publishing. https://doi.org/10.1787/b5fd1b8f-en

Palardy, G. (2013). High school socioeconomic segregation and student attainment. American Educational Research Journal, 50(4), 714-754. https://doi.org/10.3102/0002831213481240

Paulhus, D. (1991). Measurement and control of response bias. En J. Robinson, P. Shaver y L. Wrightsman (Eds.), Measures of personality and social psychological attitudes (pp. 17-59). Academic Press. https://doi.org/10.1016/B978-0-12-590241-0.50006-X

Rasbash, J., Steele, F., Browne, W. y Goldstein, H. (2012). A user's guide to MLwiN. Centre for Multilevel Modelling.

Rumberger, R. y Palardy, G. (2005). Does segregation still matter? The impact of student composition on academic achievement in high school. Teachers College Record, 107(9), 19992045. https://doi.org/10.1111/j.1467-9620.2005.00583.x

Schindler Rangvid, B. (2007). School composition effects in Denmark: Quantile regression evidence from PISA 2000. Empirical Economics, 33, 359-388.

https://doi.org/10.1007/s00181-007-0133-6

Snijders, T. y Bosker, R. (1999). Multilevel analysis. An introduction to basic and advanced multilevel modeling. Sage. 
Treviño, E., Valdés, H., Castro, M., Costilla, R., Pardo, C. y Donoso, F. (2010). Factores asociados al logro cognitivo de los estudiantes de América Latina y El Caribe. OREALC/UNESCO \& LLECE.

Van Ewijk, R. y Sleegers, P. (2010). The effect of peer socioeconomic status on student achievement: A meta-analysis. Educational Research Review, 5, 134-150.

https://doi.org/10.2139/ssrn.1402645

Vázquez, E. (2016). Segregación escolar por nivel socioeconómico. Midiendo el fenómeno y explorando sus determinantes. Económica, 2, 121-184.

Willms, J. (2003). Ten hypotheses about socioeconomic gradients and community differences in children's developmental outcomes. Canadian Research Institute for Social Policy.

Willms, D. (2006). Learning divides: Ten policy questions about the performance and equity of schools and schooling systems. UNESCO.

Willms, D. y Somers, M. (2001). Family, classroom, and school effects on children's educational outcomes in Latin America. School Effectiveness and School Improvement, 12(4), 409-445. https://doi.org/10.1076/sesi.12.4.409.3445 


\section{Anexo 1}

Cuadro 6. Regresión Multinivel. Coeficientes de todas las variables explicativas en los modelos finales: modelos trivariados

\begin{tabular}{|c|c|c|c|c|c|c|c|c|c|c|c|c|c|c|c|}
\hline & \multicolumn{3}{|c|}{ ARGENTINA } & \multicolumn{3}{|c|}{ BRASIL } & \multicolumn{3}{|c|}{ CHILE } & \multicolumn{3}{|c|}{ COLOMBIA } & \multicolumn{3}{|c|}{ Costa Rica } \\
\hline & $\mathbf{L}$ & $\mathbf{M}$ & C & $\mathbf{L}$ & $\mathbf{M}$ & $\mathbf{C}$ & $\mathbf{L}$ & $\mathbf{M}$ & C & $\mathbf{L}$ & $\mathbf{M}$ & $\mathbf{C}$ & $\mathbf{L}$ & $\mathbf{M}$ & C \\
\hline Edad & 2,9 & $8,9^{*}$ & 7,4 & $-19,7 * * * *$ & $-19,2$ **** & $-21,8 * * *$ & $-2,0$ & $3,7 *$ & $7,0^{* * * * *}$ & $7,5 * *$ & 1,1 *** & $4,0^{* * *}$ & $-13,1 * * *$ & $-16,0^{* * * *}$ & $-13,4 * * * *$ \\
\hline Mujer & 13,3 ***** & $-17,3^{* * * * *}$ & $-11,8 * * *$ & $15,2 * * *$ & $-17,8 * * *$ & $-8,2$ **** & 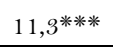 & $-15,3$ **** & $-10,0$ **** & 3,2 & $-26,4 * * * *$ & $-18,2^{2 * * *}$ & $8,2^{* * * * *}$ & $-22,4 * * * *$ & $-14,1$ ***** \\
\hline Apoyo emocional & $2,1 *$ & $0,8^{*}$ & $0,4 * * *$ & sd & sd & $\mathrm{sd}$ & 1,1 & 1,4 & 0,6 & $\mathrm{sd}$ & sd & sd & $2,7 * * * *$ & 2,1 **** & $-0,5$ \\
\hline Preescolar & $5,2^{*}$ & $4,7^{*}$ & $2,4 *$ & 1,8 & 3,9 & 1,2 & 0,7 & 1,6 & 1,5 & $4,5 *$ & $6,5^{*}$ & $3,9^{*}$ & $-1,9$ & $-2,1$ & $-0,0^{* * * *}$ \\
\hline NSE del hogar & $7,6^{* * * *}$ & $8,9^{* * * *}$ & $9,3 * * *$ & $5,4 * * * *$ & $6,6^{* * * *}$ & $6,2^{* * * * *}$ & $5,6 * * *$ & $7,2^{* * * * *}$ & $7,2^{*} * * *$ & $4,7 * * * *$ & $2,3 * * *$ & 2,7 ***** & $6,4^{*} * * *$ & $5,3 * * * *$ & $7,8 * * *$ \\
\hline Grado modal & $53,0^{* * * * *}$ & $41,9^{* * * *}$ & $49,4^{*} * * *$ & $48,6^{* * * *}$ & $40,9^{* * * *}$ & $46,6^{* * * *}$ & $54,3 * * * *$ & 48,6 ***** & $46,0^{* * * *}$ & $52,4 * * * *$ & 47,7 ***** & $46,0^{* * * *}$ & $39,1 * * * *$ & $38,6^{* * * *}$ & $35,6^{* * * *}$ \\
\hline Composición escolar & $41,2^{* * *}$ & 38,2 **** & $37,4^{* * * * *}$ & 35,2 ***** & $29,0^{* * * * *}$ & $29,4^{* * * *}$ & $41,4^{* * * *}$ & 39,3 & $36,1 * * * *$ & $38,5 * * *$ & $34,3^{*} * * *$ & $36,0^{* * * * *}$ & $39,4^{* * * *}$ & $36,0^{* * * *}$ & 34,2 **** \\
\hline Proporción de mujeres & $-34,1^{*}$ & $-28,4^{*}$ & $-32,8^{*}$ & $61,8^{* * * *}$ & $56,3 * * * *$ & 57,3 **** & $22,2^{*}$ & $25,4^{*}$ & $16,9^{*}$ & $57,9^{* *}$ & $38,2^{* * * *}$ & $49,6^{* * * * *}$ & 28,1 ***** & $25,0^{* * * *}$ & $19,0^{* * * *}$ \\
\hline Tamaño escolar & 0,0 & 0,0 & 0,0 & 0,0 & 0,0 & 0,0 & 0,0 & 0,0 & 0,0 & 0,0 & 0,0 & 0,0 & 0,0 & 0,0 & 0,0 \\
\hline Gestión privada & 10,9 & 5,2 & 5,6 & 37,7 ***** & 38,6 **** & $38,2 * * *$ & $12,4^{*}$ & $15,4^{*}$ & $12,8^{*}$ & $-2,8$ & 0,8 & $-1,7$ & $-19,0$ & $-22,3$ & $-16,7$ \\
\hline Escuela rural & $-12,1 *$ & $-8,1 *$ & $-8,5 *$ & 2,6 & 6,0 & 2,8 & 4,2 & 4,0 & 6,8 & 1,0 & 4,2 & 2,4 & $-2,9$ & $-2,7$ & 0,1 \\
\hline Agrupamiento & $-1,0$ & 3,0 & 1,8 & 4,0 & $-0,1$ & 3,2 & $-5,3$ & $-4,0$ & $-4,6$ & $-5,9$ & $-2,2$ & $-4,3$ & $11,4 * *$ & $10,8^{* * *}$ & $11,2^{* * *}$ \\
\hline Selectividad & $-4,7$ & $-3,2$ & $-2,0$ & $-8,5$ & $-3,4$ & $-6,5$ & 16,0 & 20,9 & 20,9 & $-2,9$ & $-3,0$ & $-3,3$ & 2,6 & 0,7 & 3,6 \\
\hline Recursos escasos & $-0,4$ & $-0,6$ & $-0,7$ & $-4,3^{*}$ & $-3,5^{*}$ & $-4,4^{*}$ & 0,5 & $-0,1$ & $-0,1$ & sd & sd & $\mathrm{sd}$ & $-2,3$ & $-0,9$ & $-2,1$ \\
\hline Personal escaso & $-1,2$ & $-0,2$ & $-0,0$ & $\mathrm{sd}$ & sd & sd & $-0,6$ & 0,9 & 0,3 & $-1,4$ & 0,3 & $-0,6$ & 3,0 & 1,0 & 3,0 \\
\hline Interés de docentes & $4,9^{* * * *}$ & $1,4 * *$ & $2,8 * * *$ & $3,3 * * *$ & $3,6^{* * * *}$ & $2,6^{* * * *}$ & $6,7 * * *$ & $6,5 * * * *$ & 4,8 **** & $4,8 * * *$ & 3,0 **** & $2,8 * * *$ & $4,9^{* * * *}$ & $2,7 * *$ & $3,4 * *$ \\
\hline Docentes con Maestría & $-1,7$ & 0,4 & $-3,3$ & 10,0 & 7,9 & 11,6 & $40,4^{*}$ & $29,9^{*}$ & $45,9^{*}$ & 15,4 & 26,4 & 12,6 & $13,7^{*}$ & $16,2^{*}$ & $17,0^{*}$ \\
\hline
\end{tabular}

Notas L: nota en Lectura; M: nota en Matemática; C: nota en Ciencias; *Significatividad al 10\%; **Significatividad al 5\%; ***Significatividad al $1 \%$; ns: no significativo; sd: sin datos; por problemas de cómputo, no se incluyó la variable explicativa en el modelo.

Fuente: Elaboración propia a partir de la base de datos PISA 2018 (OCDE). 
Cuadro 6. (cont.). Regresión Multinivel. Coeficientes de todas las variables explicativas en los modelos finales: modelos trivariados

\begin{tabular}{|c|c|c|c|c|c|c|c|c|c|c|c|c|c|c|c|}
\hline & \multicolumn{3}{|c|}{ MÉXICO } & \multicolumn{3}{|c|}{ PANAMÁ } & \multicolumn{3}{|c|}{ Perú } & \multicolumn{3}{|c|}{ REP. DOMINICANA } & \multicolumn{3}{|c|}{ URUGUAY } \\
\hline & $\mathbf{L}$ & $\mathbf{M}$ & $\mathbf{C}$ & $\mathbf{L}$ & M & $\mathbf{C}$ & $\mathbf{L}$ & M & $\mathbf{C}$ & $\mathbf{L}$ & $\mathbf{M}$ & C & $\mathbf{L}$ & $\mathbf{M}$ & $\mathbf{C}$ \\
\hline Edad & 5,2 & 3,8 & 7,4 & $7,1 * *$ & $8,4 * *$ & $5,8 * *$ & 0,8 & $-9,1 * *$ & $-0,6$ & 1,5 & 3,2 & 3,8 & $7,6 *$ & $9,1^{*}$ & 4,7 \\
\hline Mujer & $6,1 * * * *$ & $-17,5$ **** & $-12,3$ **** & $4,6 * *$ & $-15,5 * * * *$ & $-8,6^{* * * *}$ & $6,2 * *$ & $-21,1 * * * *$ & $-16,3 * * *$ & $15,4 * * * *$ & $-10,3$ ***** & $-2,2^{*} * * *$ & 10,9 **** & $-18,7$ ***** & $-12,7^{* * * * *}$ \\
\hline Apoyo emocional & $1,5^{*}$ & $0,2^{*}$ & 0,2 & 1,1 & $-0,7$ & $-0,5$ & 1,7 & 1,5 & $-0,1$ & $1,8^{* * *}$ & 0,2 & 0,5 & 1,5 & 1,1 & 0,1 \\
\hline Preescolar & $6,6^{* * *}$ & $13,8^{* * * *}$ & $6,8^{* * *}$ & $4,9^{* *}$ & $3,2^{* * *}$ & $6,0^{* * *}$ & $4,9^{*}$ & $7,0^{*}$ & $5,7 *$ & $5,4 * *$ & $2,9^{* * *}$ & 7,9 *** & 10,7 **** & $12,8 * * *$ & $11,9^{* * * *}$ \\
\hline NSE del hogar & $4,8^{* * * *}$ & $3,8 * * *$ & $4,7 * * *$ & $3,8^{* * * *}$ & $3,0 * * *$ & 3,3 **** & 8,0 ***** & $8,2 * * * *$ & 8,2 **** & $2,8^{* * *}$ & $3,3 * *$ & $4,2^{*} * *$ & $9,1 * * * *$ & $7,6^{* * * *}$ & $9,7 * * * *$ \\
\hline Grado modal & $30,9^{* * * *}$ & 34,4 ***** & 28,6 **** & 37,5 **** & $37,0 * * *$ & 38,0 **** & 52,8 **** & $56,9 * * *$ & $50,3 * * * *$ & $52,9^{* * * *}$ & 45,6 **** & 42,9 **** & 78,9 **** & 73,9 **** & $70,1 * * * *$ \\
\hline Composición escolar & $16,3^{*}$ & 10,1 & $16,2^{*}$ & 29,1 **** & 27,1 ***** & 26,8 **** & $35,8^{* * * *}$ & $28,0 * * *$ & $26,9^{* * * *}$ & $28,9^{* * * *}$ & $25,4 * * * *$ & $22,5 * * * *$ & 31,5 **** & 28,6 **** & $25,6^{* * * *}$ \\
\hline Proporción de mujeres & 6,2 & 11,5 & 7,4 & 14,8 & 7,0 & 4,8 & 8,8 & 4,1 & 1,8 & $42,4 * * * *$ & $35,7 * * * *$ & $19,1 * * *$ & $29,1^{*}$ & 23,2 & 3,7 \\
\hline Tamaño escolar & $0,0 * *$ & 0,0 & 0,0 & 0,0 & 0,0 & 0,0 & 0,0 & 0,0 & 0,0 & 0,0 & 0,0 & 0,0 & 0,0 & 0,0 & 0,0 \\
\hline Gestión privada & 15,7 & 7,0 & 5,0 & 8,1 & 7,7 & 17,7 & 13,3 & 12,0 & 10,6 & 4,3 & 5,2 & 6,3 & $-15,0$ & $-7,9$ & $-6,3$ \\
\hline Escuela rural & $-9,6$ & $-7,1$ & $-2,6$ & $-3,3$ & $-3,0$ & 2,3 & 11,4 & 8,2 & 9,3 & $-10,8$ & $-9,1$ & $-9,2$ & $-7,2$ & $-5,9$ & $-1,9$ \\
\hline Agrupamiento & $-7,8$ & $-3,0$ & $-5,9$ & $-7,6^{* *}$ & $-4,8 * *$ & $-4,2 * *$ & $-16,1 * *$ & $-8,7^{*}$ & $-11,3 * *$ & 3,1 & 4,3 & 2,5 & 0,2 & 0,3 & $-0,9$ \\
\hline Selectividad & $-4,7$ & $-2,3$ & $-7,1$ & 2,4 & 1,9 & 1,7 & $-8,6$ & $-3,4$ & $-6,5$ & $-1,4$ & $-1,1$ & $-0,8$ & 1,7 & 1,2 & $-0,3$ \\
\hline Recursos escasos & 1,7 & $-2,3$ & 1,9 & $-3,6$ & $-1,6$ & $-1,7$ & $-3,7$ & $-2,6$ & $-2,7$ & $-0,4$ & 0,9 & 0,5 & 1,6 & 0,8 & 0,7 \\
\hline Personal escaso & $-8,9^{*}$ & $-5,4$ & $-8,7^{*}$ & 1,6 & 0,7 & 1,3 & 2,7 & 3,2 & 2,1 & $-0,9$ & $-1,1$ & $-1,0$ & $-2,7$ & $-2,6$ & $-2,8$ \\
\hline Interés de docentes & $3,1 * * * *$ & $1,8 * * * *$ & $3,1 * * *$ & 0,0 & 0,8 & $-0,9$ & 1,3 & 1,9 & 0,9 & $3,8 * * * *$ & $2,5 * * * *$ & 4,7 ***** & $4,9^{* * * *}$ & $1,3^{*}$ & $5,1 * * *$ \\
\hline Docentes con Maestría & $45,2^{*} * * *$ & 57,1 ***** & $47,2^{*} * * *$ & 9,7 & 4,1 & 11,4 & 8,0 & 15,2 & 3,2 & $-8,4$ & $-3,8$ & $-11,3$ & $-94,9$ & $-36,3$ & $-82,5$ \\
\hline
\end{tabular}

Notas: L: nota en Lectura; M: nota en Matemática; C: nota en Ciencias; *Significatividad al 10\%; **Significatividad al 5\%; ***Significatividad al 1\%.; ns: no significativo; sd: sin datos; por problemas de cómputo, no se incluyó la variable explicativa en el modelo.

Fuente: Elaboración propia a partir de la base de datos PISA 2018 (OCDE). 
Cuadro 7. Regresión Multinivel. Coeficientes de todas las variables explicativas en los modelos finales: modelos bivariados

\begin{tabular}{|c|c|c|c|c|c|c|c|c|c|c|}
\hline & \multicolumn{2}{|c|}{ ARgENTINA } & \multicolumn{2}{|c|}{ BRASIL } & \multicolumn{2}{|c|}{ CHILE } & \multicolumn{2}{|c|}{ COLOMBIA } & \multicolumn{2}{|c|}{ Costa RicA } \\
\hline & $\begin{array}{l}\text { Nota en } \\
\text { Lectura } \\
\end{array}$ & $\begin{array}{l}\text { Exp. } \\
\text { Ocup. }\end{array}$ & $\begin{array}{l}\text { Nota en } \\
\text { Lectura } \\
\end{array}$ & $\begin{array}{l}\text { Exp. } \\
\text { Ocup. }\end{array}$ & $\begin{array}{l}\text { Nota en } \\
\text { Lectura } \\
\end{array}$ & $\begin{array}{l}\text { Exp. } \\
\text { Ocup. }\end{array}$ & $\begin{array}{l}\text { Nota en } \\
\text { Lectura } \\
\end{array}$ & $\begin{array}{l}\text { Exp. } \\
\text { Ocup. }\end{array}$ & $\begin{array}{l}\text { Nota en } \\
\text { Lectura }\end{array}$ & $\begin{array}{l}\text { Exp. } \\
\text { Ocup. }\end{array}$ \\
\hline Edad & $3,1^{* *}$ & $1,8^{* *}$ & $-21,1 * * *$ & $-1,6^{*}$ & $-2,6$ & $1,4^{*}$ & $7,5^{* *}$ & $-0,2$ & $-14,1 * * * *$ & $-1,3^{*}$ \\
\hline Mujer & $12,5^{* * *}$ & $6,6^{* * * *}$ & $15,8^{* * * *}$ & $7,6^{* * *}$ & $10,6^{* * *}$ & $5,8^{* * *}$ & 3,1 & $4,3^{* * * *}$ & $8,1^{* * *}$ & $4,5 * * *$ \\
\hline Apoyo emocional & $2,0^{* * *}$ & 0,0 & sd & sd & 0,9 & $-0,0$ & 1,1 & 0,4 & $2,6^{* * *}$ & 0,3 \\
\hline Preescolar & $5,4 * *$ & $-0,3$ & 1,0 & $-0,2$ & 1,2 & 0,6 & $4,8^{* *}$ & $-0,2$ & $-1,3$ & 0,6 \\
\hline NSE del hogar & 7,7 *** & $1,4^{* * * *}$ & $5,3^{* * *}$ & $0,7 * * *$ & $5, \mathrm{O}^{* *}$ & $1,6^{* * *}$ & $4,1^{* * *}$ & $0,6^{* * *}$ & $6,5^{* * *}$ & $0,4^{* *}$ \\
\hline Grado modal & $53,7^{* * * *}$ & $3,9^{* * *}$ & $50,2^{* * * *}$ & $2,2^{* * * *}$ & $54,7 * * *$ & $4,0^{* * * *}$ & $53,0^{* * * *}$ & $2,3^{* * *}$ & $40,3^{* * * *}$ & 1,7 *** \\
\hline Composición escolar & $42,3 * * *$ & $1,1^{*}$ & $36,7 * * *$ & $1,2^{*}$ & $41,5 * * *$ & $3,8^{* * * *}$ & 39,7 **** & $1,1 * *$ & $39,1 * * *$ & $2,2 * * *$ \\
\hline Proporción de mujeres & $-31,0^{*}$ & 1,8 & $59,4^{* * * *}$ & 0,7 & 13,3 & 3,9 & $54,2^{* *}$ & $4,1^{* *}$ & 28,9 & 0,1 \\
\hline Tamaño escolar & 0,0 & 0,0 & 0,0 & 0,0 & 0,0 & $0,0^{*}$ & 0,0 & 0,0 & 0,0 & 0,0 \\
\hline Gestión privada & 8,6 & 1,5 & 34,7 **** & $2,4^{* * * *}$ & 10,2 & $2,1^{* * *}$ & $-1,7$ & $-1,0$ & $-16,7$ & $-1,7^{*}$ \\
\hline Escuela rural & $-12,5^{* *}$ & 0,7 & 6,0 & $-0,3$ & 3,7 & 0,4 & 4,1 & $-0,2$ & $-2,8$ & $-0,0$ \\
\hline Agrupamiento & $-1,1$ & $-0,2$ & 4,1 & 0,2 & $-0,9$ & $-0,4$ & $-0,7$ & $-0,5$ & $11,9^{* *}$ & $-0,6$ \\
\hline Selectividad & $-1,6$ & $-0,8$ & $-6,3$ & 0,9 & $14,2^{* * *}$ & 0,1 & $-3,2$ & 0,1 & 1,7 & 0,4 \\
\hline Recursos escasos & 1,7 & $-0,4$ & $\mathrm{Sd}$ & $\mathrm{Sd}$ & $-1,0$ & 0,4 & $-1,3$ & 0,2 & $-1,9$ & $-0,7^{* * * *}$ \\
\hline Personal escaso & $-4,3^{*}$ & 0,2 & 4,0 & $-0,3$ & $-1,1$ & $-0,3$ & $-0,1$ & 0,0 & 2,9 & 0,1 \\
\hline Interés de docentes & $5,2^{* * *}$ & 0,3 & $3,6^{* * * *}$ & 0,3 & $7,0^{* * * *}$ & $0,8^{* * *}$ & $4,6^{* * * *}$ & 0,3 & $5,0^{* * * *}$ & 0,2 \\
\hline Docentes con Maestría & $-0,3$ & $-1,2$ & $28,1 * *$ & $-2,1$ & $51,8 * * *$ & 2,1 & 6,4 & $-3,8^{*}$ & 11,1 & 1,4 \\
\hline
\end{tabular}

Notas: L: nota en Lectura; M: nota en Matemática; C: nota en Ciencias; *Significatividad al 10\%; **Significatividad al 5\%; ***Significatividad al 1\%.; ns: no significativo; sd: sin datos; por problemas de cómputo, no se incluyó la variable explicativa en el modelo.

Fuente: Elaboración propia a partir de la base de datos PISA 2018 (OCDE). 
Cuadro 7 (cont.). Regresión Multinivel. Coeficientes de todas las variables explicativas en los modelos finales: modelos bivariados

\begin{tabular}{|c|c|c|c|c|c|c|c|c|c|c|}
\hline & \multicolumn{2}{|c|}{ MÉXICO } & \multicolumn{2}{|c|}{ PANAMá } & \multicolumn{2}{|c|}{ PERÚ } & \multicolumn{2}{|c|}{ REP. DOMINICANA } & \multicolumn{2}{|c|}{ URUGUAY } \\
\hline & $\begin{array}{l}\text { Nota en } \\
\text { Lectura }\end{array}$ & $\begin{array}{l}\text { Exp. } \\
\text { Ocup. }\end{array}$ & $\begin{array}{l}\text { Nota en } \\
\text { Lectura }\end{array}$ & $\begin{array}{l}\text { Exp. } \\
\text { Ocup. }\end{array}$ & $\begin{array}{l}\text { Nota en } \\
\text { Lectura }\end{array}$ & $\begin{array}{l}\text { Exp. } \\
\text { Ocup. }\end{array}$ & $\begin{array}{l}\text { Nota en } \\
\text { Lectura }\end{array}$ & $\begin{array}{l}\text { Exp. } \\
\text { Ocup. }\end{array}$ & $\begin{array}{l}\text { Nota en } \\
\text { Lectura }\end{array}$ & $\begin{array}{l}\text { Exp. } \\
\text { Ocup. }\end{array}$ \\
\hline Edad & $6,4^{*}$ & 0,6 & $8,1 * *$ & $-0,1$ & 1,7 & $-0,5$ & 1,6 & $-1,5$ & $7,9^{*}$ & 0,2 \\
\hline Mujer & $5,5 * * *$ & $2,1 * * *$ & $4,2 * * *$ & $1,3 * * *$ & $5,9^{* * * *}$ & $4,2 * * *$ & $15,5^{* * * *}$ & $4,2^{* * * *}$ & $11, \mathrm{O}^{* * * *}$ & $8,8 * * *$ \\
\hline Apoyo emocional & $1,7 *$ & $-0,1$ & 1,3 & 0,5 & $1,6^{*}$ & $0,5^{*}$ & $1,9 * *$ & $-0,1$ & 1,6 & $0,5^{*}$ \\
\hline Preescolar & 6,0 & 0,8 & $4,6^{* * *}$ & $-0,4$ & 4,6 & 0,6 & $5,3^{* *}$ & 0,8 & $10,4^{* * *}$ & $2,3^{* *}$ \\
\hline NSE del hogar & 4,9 **** & $0,9 * * *$ & $3,9^{* * *}$ & 0,3 & $8,1 * * *$ & $1,4 * * *$ & $2,5^{* *}$ & 0,0 & $9,3 * * *$ & $1,4 * * *$ \\
\hline Grado modal & $31,2^{* * * *}$ & $4,4 * * *$ & $38,2 * * *$ & $3,8 * * *$ & $54, \mathrm{O}^{* * * *}$ & $4,0^{* * * *}$ & $52,4 * * *$ & 0,2 & $77,2^{*} * * *$ & $9,4 * * *$ \\
\hline Composición escolar & $17,2^{* *}$ & 0,2 & $29,6^{* * * *}$ & $-0,3$ & 33,7 **** & 1,1 & 29,9 *** & 0,9 & $31,9^{* * * *}$ & $3,5^{\text {**** }}$ \\
\hline Proporción de mujeres & 3,2 & $-5,5 * *$ & 17,1 & 2,6 & 2,2 & $-0,0$ & 39,9 *** & $-0,7$ & $27,7^{*}$ & $5,4^{*}$ \\
\hline Tamaño escolar & 0,0 & 0,0 & 0,0 & 0,0 & 0,0 & 0,0 & 0,0 & $0,0^{* *}$ & 0,0 & 0,0 \\
\hline Gestión privada & 9,5 & 1,0 & 6,9 & 2,0 & 10,4 & 0,2 & 1,8 & $-0,8$ & $-13,6$ & $-1,3$ \\
\hline Escuela rural & $-10,2$ & $-1,5$ & $-4,7$ & 0,5 & 5,1 & 0,1 & $-12,8^{* *}$ & $1,5 * *$ & $-6,1$ & 0,1 \\
\hline Agrupamiento & $-6,6$ & 0,2 & $-5,1$ & $-0,8$ & $-12,9^{* *}$ & $-2,0^{*}$ & 3,0 & $-0,6$ & $-0,2$ & $1,7^{*}$ \\
\hline Selectividad & $-2,8$ & 1,1 & 2,3 & $-0,4$ & $-3,6$ & $-2,1$ & 0,9 & 0,1 & 2,8 & $-1,0$ \\
\hline Recursos escasos & $-0,4$ & 0,1 & $-4,6^{*}$ & 0,1 & $-4,0$ & 0,0 & $-1,5$ & $-0,5$ & 1,2 & $-0,3$ \\
\hline Personal escaso & $-7,5$ & $-0,5$ & 1,1 & $-1,0^{*}$ & 1,8 & $-0,1$ & 0,3 & 0,2 & $-2,2$ & $-0,1$ \\
\hline Interés de docentes & $2,9^{* *}$ & 0,3 & 0,2 & 0,1 & 1,2 & 0,3 & $3,9^{* * * *}$ & 0,1 & $5,1^{* * * *}$ & 0,4 \\
\hline Docentes con Maestría & 47,3 **** & $-3,4$ & 1,1 & 1,2 & 12,3 & $-2,8$ & $-2,9$ & $-4,1^{* * *}$ & $-98,4$ & $-14,7$ \\
\hline
\end{tabular}

Notas: L: nota en Lectura; M: nota en Matemática; C: nota en Ciencias; *Significatividad al 10\%; **Significatividad al 5\%; ***Significatividad al $1 \%$; ns: no significativo; sd: sin datos; por problemas de cómputo, no se incluyó la variable explicativa en el modelo.

Fuente: Elaboración propia a partir de la base de datos PISA 2018 (OCDE). 


\section{Anexo 2: Ecuaciones correspondientes a los modelos estimados}

\section{Modelo multinivel univariado}

Modelo nulo

Nivel 1 (alumnos):

$$
Y_{i j}=\pi_{0 j}+e_{i j}
$$

Nivel 2 (escuelas):

$$
\pi_{0 j}=\beta_{00}+r_{0 j}
$$

Donde:

$\left.Y_{i j}\right)$ : resultado esperado del alumno i que asiste a la escuela j-

$\left(\pi_{0 j}\right)$ : logro promedio para la escuela ${ }^{j}$; y $\left({ }^{e_{i j}}\right)$ : desviación aleatoria del puntaje del alumno i con respecto al promedio de su escuela. Se asume una distribución normal con media cero y varianza constante.

$\left.{ }_{(} \beta_{00}\right)$ : media global para todas las escuelas; y $\left({ }^{r_{0 j}}\right)$ : desviación aleatoria del promedio de la escuela $j$ con respecto a la media global. Se asume una distribución normal con media cero y varianza constante.

Modelo completo con variables explicativas:

$$
Y_{i j}=\beta_{00}+\sum_{p=1}^{P} \pi_{p 0} X_{p i j}+\sum_{q=1}^{Q} \beta_{q} Z_{q j}+e_{i j}+r_{0 j}
$$

Donde $\mathrm{X}$ es un vector de $\mathrm{P}$ factores personales y familiares y $\mathrm{Z}$ representa un vector de $\mathrm{Q}$ factores escolares con efectos fijos.

\section{Modelo multinivel multivariado}

El conjunto de variables dependientes conforma un nuevo nivel por debajo del nivel de alumnos. Es decir que, los distintos logros o variables-respuesta (actual nivel 1) se anidan dentro de los alumnos (actual nivel 2), los cuales se anidan dentro de las escuelas (actual nivel 3).

Modelo completo multivariado con variables explicativas:

$$
\mathrm{Y}_{\mathrm{ij}}=\sum_{\mathrm{k}=1}^{\mathrm{K}} \omega_{\mathrm{k}}\left(\beta_{\mathrm{h} 00}+\sum_{\mathrm{p}=1}^{\mathrm{P}} \pi_{\mathrm{hp} 0} \mathrm{X}_{\mathrm{pij}}+\sum_{\mathrm{q}=1}^{\mathrm{Q}} \beta_{\mathrm{hq}} \mathrm{Z}_{\mathrm{qj}}+\mathrm{e}_{\mathrm{hij}}+\mathrm{r}_{\mathrm{h} 0 \mathrm{j}}\right)
$$

Donde:

(h): indica la variable-respuesta presente en la estimación. Tanto k como h toman valores 1 y 2 en los modelos bivariados; y 1, 2 y 3 en los modelos trivariados.

$\omega_{\mathrm{k}}=1$ si $\mathrm{k}=\mathrm{h} ;$ o si $\mathrm{k} \neq \mathrm{h}$ 


\section{Breve CV de la autora}

\section{Natalia Krüger}

Investigadora Adjunta del Consejo Nacional de Investigaciones Científicas y Técnicas (CONICET) y Profesora Adjunta del Departamento de Economía de la Universidad Nacional del Sur (UNS), Bahía Blanca, Argentina. Es Doctora en Economía por la UNS y ha realizado estancias de investigación doctoral y postdoctoral en la Universidad de Barcelona, España, la Universidade Federal Fluminense, Brasil, y la Katholieke Universiteit Leuven, Bélgica. Investiga en el área de Economía de la Educación, y sus publicaciones se enfocan en temas relativos a la calidad y la igualdad de oportunidades educativas en América Latina. ORCID ID: https://orcid.org/0000-0001-84012639. Email: natalia.kruger@uns.edu.ar 